\title{
New measurements on isobaric fission product yields and mean kinetic energy for ${ }^{241} \mathrm{Pu}$ thermal neutron-induced fission
}

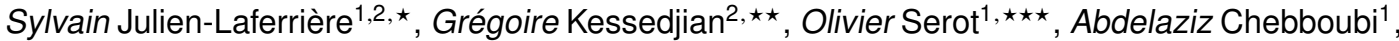 \\ David Bernard ${ }^{1}$, Aurélien Blanc ${ }^{3}$, Ulli Köster ${ }^{3}$, Olivier Litaize ${ }^{1}$, Thomas Materna ${ }^{4}$, Olivier Meplan², \\ Michal Rapala ${ }^{4}$, and Christophe Sage ${ }^{2}$ \\ ${ }^{1} C E A, D E N, D E R, S P R C, 13108$ Saint-Paul-Lez-Durance, France \\ ${ }^{2}$ LPSC, Université Grenoble-Alpes, CNRS/IN2P3, 38026 Grenoble, France \\ ${ }^{3} / L L, 38042$ Grenoble, France \\ ${ }^{4}$ CEA, DSM, IRFU, SPhN, 91191 Gif-sur-Yvette, France
}

\begin{abstract}
Nuclear fission yields data measurements for thermal neutron induced fission of ${ }^{241} \mathrm{Pu}$ have been carried out at the Institut Laue Langevin (ILL) in Grenoble, using the Lohengrin mass spectrometer. Mass, isotopic and isomeric yields have been extracted for the last measurements. A focus is given in this document to the mass yield results which are obtained for almost the entire heavy peak and most of the light high yields masses, along with the covariance matrix. The mean kinetic energy as a function of the fission product mass has also been extracted from the measurements. The total mean kinetic energy pre and post neutron emission have been assessed and compared to other works showing a rather good agreement.
\end{abstract}

\section{Introduction}

The isobaric fission product yield, or mass yield $Y(A)$, is the production rate by fission of a nucleus of mass $A$ including all the possible nuclear charge $Z$. The fission product yield knowledge for thermal neutron induced fission, condensed in the recent evaluated nuclear data libraries (JEFF-3.1.1, ENDF/B-VII.1, JENDL-4.0 ...), is one of the major contributor to the uncertainty on computed reactor physics quantities (see for example [1]). Furthermore, the uncertainty propagation can not be properly done since the yield variance-covariance matrices are missing from the evaluated data libraries, despite recent efforts to address this issue [2-5]. Additionally, some discrepancies between the major libraries exist and need to be understood. For these reasons, a collaboration of the LPSC, the CEA and the ILL focuses on producing precise measurements of fission yields for major actinides with their related experimental variance-covariance matrice [6]. In this document, the ${ }^{241} \mathrm{Pu}$ thermal neutron induced fission isobaric yields and fission product kinetic energy distribution as function of the fission product mass will be addressed. The experimental setup will be presented in Sect. 2, followed by the analysis procedure to extract the isobaric yields in Sect. 3 along with the experimental

\footnotetext{
^e-mail: sylvain.julien-laferriere@cea.fr

$\star \star$ e-mail: kessedjian@lpsc.in2p3.fr

$\star \star \star$ e-mail: olivier. serot@cea.fr
} 


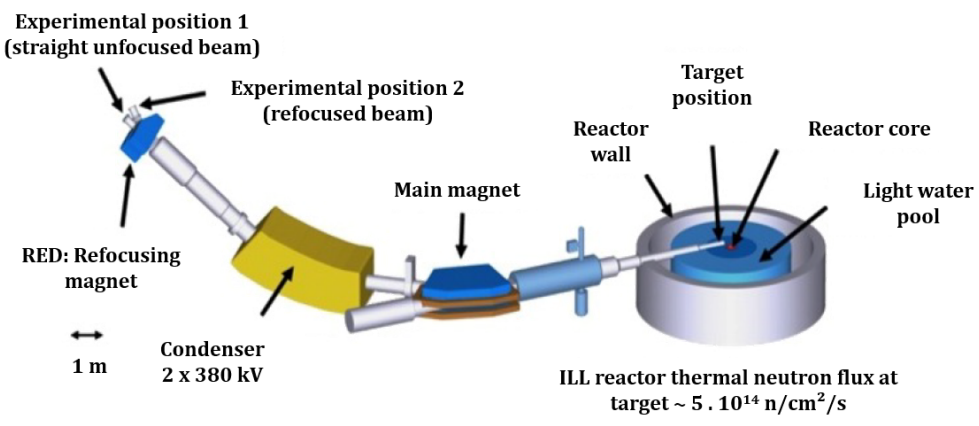

Figure 1. The Lohengrin mass spectrometer at the ILL, Grenoble. The ILL high neutron flux compensates the small geometrical acceptance of the instrument.

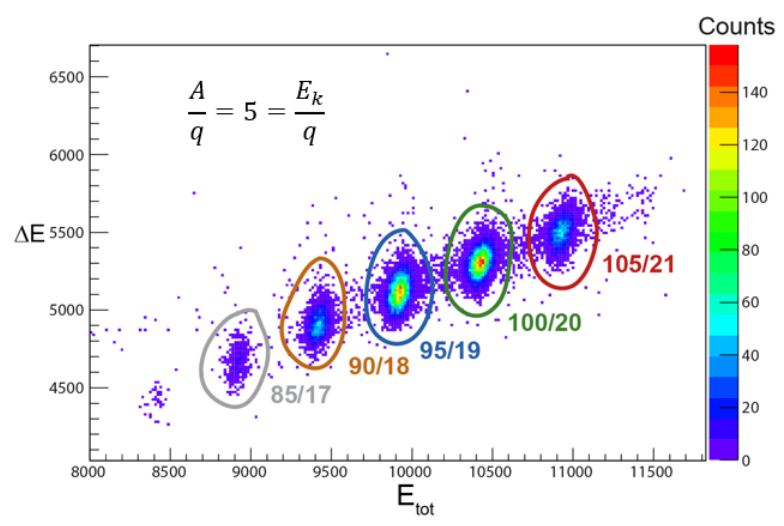

Figure 2. Raw results from the ionization chamber for the ratio $A / q=5$ and $E_{k} / q=5$. The total count rate for each mass can be measured unambiguously with sufficient statistics.

results obtained. Finally, the mean kinetic energy as a function of the fission product mass will be displayed and the corrections applied to get the mean kinetic energy before neutron emission will be detailed in Sect. 4.

\section{Experimental setup}

The Lohengrin recoil mass spectrometer [7, 8] of which a descriptive figure is presented Fig. 1, has been built in the 70's at the ILL and is since a major instrument for nuclear data measurements in particular due to its high mass resolution $(\triangle A / A \sim 1 / 400)$. A ${ }^{241} \mathrm{Pu}$ target is placed close to the reactor core under a high thermal neutron flux $\left(\sim 5 \cdot 10^{14} \mathrm{n} / \mathrm{s} / \mathrm{cm}^{2}\right.$ at target position) and undergoes a significant amount of fissions. Fission products which enter in the apparatus (solid angle $\leq 3.2 \cdot 10^{-5} \mathrm{sr}$ ) are deflected through a magnetic and an electric fields. The ion beam is selected according to the $A / q$ and $E_{k} / q$ ratios, where $A$ is the fission product mass, $E_{k}$ its kinetic energy and $q$ its ionic charge. The ratios $A / q$ and $E_{k} / q$ can be achieved with different triplet $\left(A, E_{k}, q\right)$ leading to a possible degeneracy. Two experimental positions exist, at position 1 , the ion beam directly enters in an ionization chamber. This position is used to measure isobaric yields, $Y(A)$. A double ionization chamber with a Frisch grid is used, giving access to a bidimentional map of the deposited energy as it can be seen Fig. 2 . From Fig. 2 one can also see that the previous degeneracy is well resolved, since each spot can be assigned to a single mass unambiguously. At position 2, the ion beam is refocused and implanted on a moving tape. Two clovers of four high purity germanium crystals each and two broad energy germanium detectors are placed in the vicinity of the tape and detect $\gamma$-rays resulting from the fission products $\beta^{-}$disintegration, allowing to measure isotopic yields $Y(A, Z)$. 


\section{Isobaric yields}

Since a beam is selected with Lohengrin according to the mass $A$, the ionic charge $q$ and the kinetic energy $E_{k}$ of the fission product, one can measure with the ionization chamber the count rate $N\left(A, E_{k}, q\right)$. The proper definition of the total mass count rate is then:

$$
N(A)=\int_{E_{k}} \sum_{q} N\left(A, E_{k}, q\right) d E_{k}
$$

Since the mass yields are normalized to 1 for the heavy peak (H), the yield of the mass $A_{i}$ will be:

$$
Y\left(A_{i}\right)=\frac{N\left(A_{i}\right)}{\sum_{j \in H} N\left(A_{j}\right)}
$$

\subsection{Analysis procedure}

Due to a limited beam time, it is not possible to measure the entire kinetic energy distribution for every ionic charge state. Thus, the measurements have to be optimised in order to avoid biases while performing as much scans as possible in a limited time. For each mass, at least three scans of the kinetic energy distribution at three different ionic charges $q_{r e f}$ and one scan of the ionic charge distribution at $E_{r e f}$ are made. A minimum of three energy scans are needed in order to estimate the correlation between the mean kinetic energy and the ionic charge (strongly dependent on the target) and be able to correct the ionic charge distribution of the energy bias. In addition, the time evolution of the fissile material quantity remaining in the target, so called Burn-Up (BU), see Fig. 3, is carefully estimated by the overall ionic charge and kinetic energy measurement of mass 136, in order to normalize each measurement. For each energy scan, Eq. (1) becomes:

$$
N(A)=\frac{\sum_{E_{k}} N\left(A, E_{k}, q_{r e f}\right)}{B U(t) P\left(q_{r e f}\right)}
$$

Therefore, at least three evaluations of the same $N(A)$ are made. When these evaluations are compatible, a mean value taking into account the experimental correlations is extracted with a reduced variance [9]:

$$
\begin{array}{r}
\overline{N(A)}=\left(\sum_{i, j}^{n, n}\left(C^{-1}\right)_{i, j}\right)^{-1}\left(\sum_{i, j}^{n, n}\left(C^{-1}\right)_{i, j} \cdot N_{j}(A)\right) \\
\operatorname{var}(\overline{N(A)})=\left(\sum_{i, j}^{n, n}\left(C^{-1}\right)_{i, j}\right)^{-1}
\end{array}
$$

Where $C$ is the experimental covariance matrix between the $N(A)$ 's, $n$ being the number of energy scans. For the very low yields in the symmetric and very asymmetric regions a different procedure has to be implemented due to the very low count rates, but will not be detailed in this document.

The mass yields $Y(A)$ are finally obtained by normalizing the heavy peak to 1 . This autonormalization gives access to absolute yields while assuring independence towards other existing data. Figure 3 shows on the right the results obtained with Lohengrin compared to JEFF-3.1.1. While important discrepancies are visible in the very low yield regions, the agreement is within the uncertainties for the rest of the distribution. Yet, a systematic difference seems to be visible for the light region, a narrower peak is obtained for the Lohengrin measurements resulting in higher yields than 

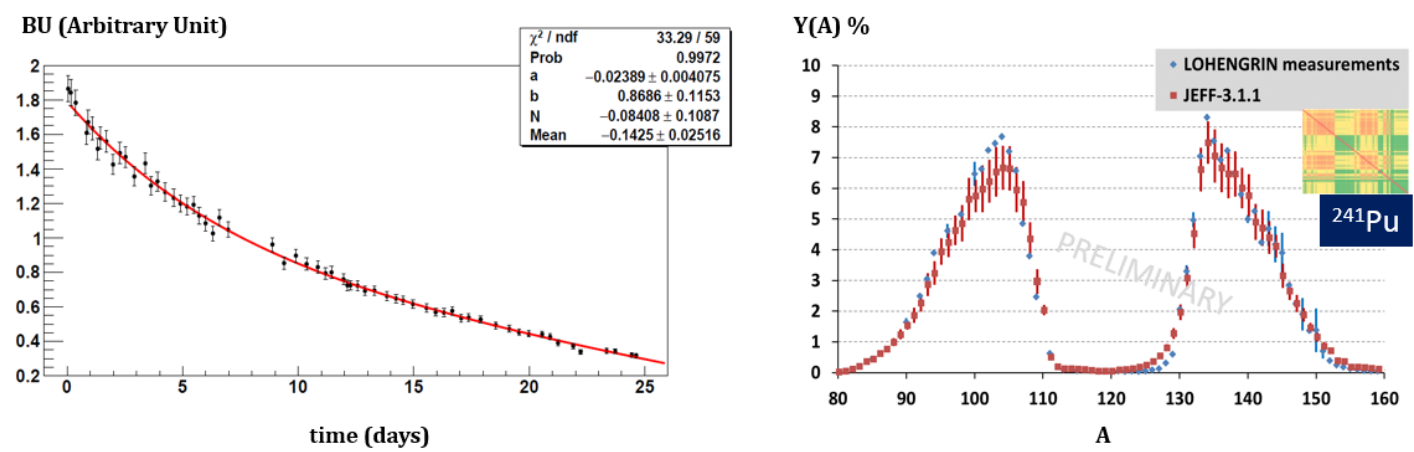

Figure 3. On the left, the time evolution of the fissile material quantity in the target, so-called Burn-Up (BU). On the right, the ${ }^{241} \mathrm{Pu}$ isobaric fission yields measured at Lohengrin (in blue), and the experimental covariance matrix, compared to JEFF-3.1.1 (in red). Lohengrin measurements are normalized to 1 for the heavy peak. These results are combining two experiments, the first one performed in 2013 [10] and the second one in 2015 [11].

JEFF-3.1.1. Since for ${ }^{241} \mathrm{Pu}$ JEFF-3.1.1 is mainly based on $2 \mathrm{E}-2 \mathrm{v}$ measurements, a possible interpretation of these differences could be a mass resolution effect not perfectly solved.

The uncertainties obtained in this work are taking into account both statistical and systematics uncertainties and are of a few percent for most of the masses and smaller than JEFF-3.1.1 uncertainties.

\section{Mean kinetic energy}

Two corrections have to be carried out in order to reach the post neutron mean kinetic energy of the fission products. First of all, since the target is evolving through time, so is the mean kinetic energy of the fission products exiting the target. As it can be seen on the left of Fig. 4, the mean of the kinetic energy distribution of mass 136 at ionic charge 23, which is the image of the $\mathrm{BU}$, is decreasing over time. This is mainly because a significant amount of fissile material is implanted on the target support over time, further away from the exit of the target facing Lohengrin. The fission products will then have a bigger mean path through the target, losing more kinetic energy in the process. The Fig. 4 , on the right, shows the correction of the measured mean kinetic energies depending on the time at which the measurement has been done The time reference is the beginning of the experiment when the target width is known. This is important since the second correction is to estimate the energy loss in the target. Two layers have to be considered for one target: the Pu layer and the protective Ni foil covering it.

For the energy loss in the Ni foil, the mean energy loss of the mass $A$ is taken to be:

$$
\frac{\overline{d E}}{d x}(A) \cdot e \cdot \rho_{\text {mat }}=3.07 \cdot 10^{-4} \cdot \frac{Z_{\text {mat }} Z_{e f f}^{2}(A)}{A_{\text {mat }} \beta^{2}(A)} L(A)
$$

Where $Z_{\text {eff }}$ is the average equilibrium charge state, $Z_{\text {mat }}$ and $A_{\text {mat }}$ the nuclear charge and mass of the penetrated material, $e$ its thickness, $\rho_{\text {mat }}$ its density, $\beta$ the reduced fission fragment velocity and $L$ the stopping number. Additional details can be found in [12]. This correction is used for the Ni foil since the needed parameters are given in [12]. The Ni foil thickness of $0.25 \mu \mathrm{m}$ is given by the constructor with a $20 \%$ uncertainty. It is assumed to be by far the major component of the uncertainty for this energy loss. 

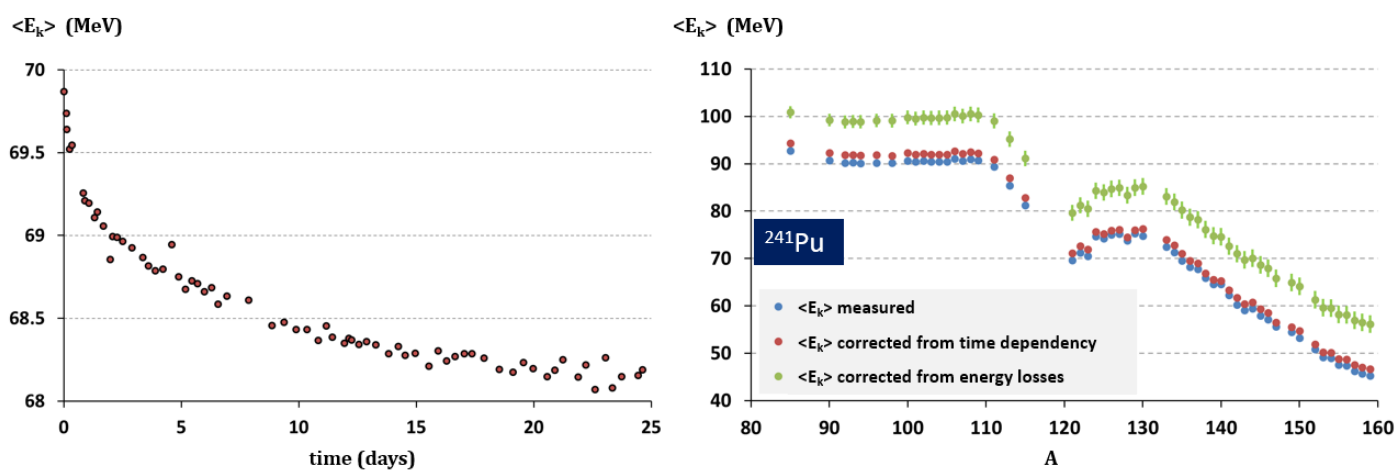

Figure 4. On the left, the evolution over time of the measured post-neutron mean kinetic energy for the mass 136 at the ionic charge 23. On the right, the measured post-neutron mean kinetic energy as a function of the fission product mass before (in blue) and after correction of the energy dependency to time (in red) and after correction from the energy loss in the $\mathrm{Pu}$ and the Ni protective foil (in green).

To estimate the kinetic energy loss in the Pu layer, the stopping range tables from the SRIM software [13] have been used. Some discrepancies have been observed in the kinetic energy loss estimation for the thin Ni layer between SRIM and the correction described in Eq. (5). For this reason, the uncertainty on the loss in the Pu layer is taken equals to be $100 \%$. In fine, the total energy loss (Ni foil + Pu layer) increases from $8 \mathrm{MeV}$ for the light masses up to $11 \mathrm{MeV}$ for the heavy masses, whose around $0.2 \mathrm{MeV}$ are lost in the Pu layer and the rest in the Ni foil, see Fig. 4.

The pre-neutron emission mean kinetic energy can also be accessed using the Monte Carlo deexcitation code Fifrelin [14] sawtooth, $\bar{v}_{F i f}(A)$, and using the following formula [15]:

$$
\frac{\bar{E}_{k-p r e}(A)-\bar{E}_{k-p o s t}(A)}{\bar{E}_{k-p r e}(A)}=\frac{\bar{v}_{F i f}(A)}{A}
$$

The Tab. 1 regroups the results obtained when averaging the mean kinetic energies for the light and heavy masses, respectively $\left\langle K E_{L}\right\rangle$ and $\left\langle K E_{H}\right\rangle$, and the total mean kinetic energy $\langle T K E\rangle$. These three quantities computed for post and pre-neutron emission are compared to existing results. This work presents higher values than previous work but consistent within the uncertainties.

A significant part of the light region has not been measured. As a consequence, the mean kinetic energy of the light fragments, $\left\langle K E_{L}\right\rangle=\sum_{i \in L} Y\left(A_{i}\right) \cdot\left\langle E_{k}\left(A_{i}\right)\right\rangle$, can not be properly computed. However, since most of the high yields have been covered, and the mean kinetic energy is not fluctuating much for the light masses, it is still a good estimation.

\section{Conclusion}

The mass yields for the entire heavy peak and a significant part of the light peak have been measured at Lohengrin and the experimental correlation matrix has been computed. The possibility to normalize the measurements without using nuclear data libraries is an important feature of this work, guaranteeing the independence of the produced fission yields to existing libraries. Further details about the estimations of the correlations between the different $\mathrm{Y}(\mathrm{A})$ and the analysis scheme in the particular case of the very low yield regions will be explicated in future publications. The symmetry region 
Table 1. Mean kinetic energy for the light fragments, $\left\langle K E_{L}>\right.$, the heavy fragments, $\left\langle K E_{H}>\right.$ and the total mean kinetic energy $<T K E>$ obtained in this work for post and pre neutron emission, compared to previous works and to JEFF-3.1.1.

\begin{tabular}{c|lcc}
\hline Post neutron emission & This work & Thierens et al. [16] & JEFF-3.1.1 \\
\hline$<K E_{L}>$ & $99.7 \pm 0.9$ & - & - \\
$<K E_{H}>$ & $75.6 \pm 1.1$ & - & - \\
$<T K E>$ & $175.8 \pm 1.5$ & $176.93 \pm 0.06$ & $175.37 \pm 0.68$ \\
& & & \\
\hline Pre neutron emission & This work & Neiler et al. [17] & \\
\hline$<K E_{L}>$ & $101.3 \pm 0.9$ & $103.2 \pm 1.0$ & \\
$<K E_{H}>$ & $76.1 \pm 1.2$ & $76.3 \pm 0.8$ & \\
$<T K E>$ & $177.5 \pm 1.6$ & $179.6 \pm 1.8$ &
\end{tabular}

is particularly interesting since it is a sensitive region to probe fission mode signature in the kinetic energy distribution, this work is still on going. The mean kinetic energies obtained are similar to previous works, especially before neutron emission correction.

This work was supported by CEA, IN2P3 and "le défi NEEDS". The authors are grateful for the support of the ILL and all the staff involved from CEA Cadarache and LPSC.

\section{References}

[1] N. Terranova, PhD thesis, Covariance Evaluation for Nuclear Data of Interest to the Reactivity Loss Estimation of the Jules Horowitz Material Testing Reactor (Université di Bologna, 2016) Chapter 6

[2] N. Terranova, O. Serot, P. Archier, C. de Saint Jean and M. Sumini, Nucl. Data Sheets 123, 225-230 (2015)

[3] N. Terranova, O. Serot, P. Archier, C. de Saint Jean and M. Sumini, accepted for publication in Annals of Nucl. En. (2017)

[4] L. Fiorito, A. Stankovskiy, G. Van den Eynde, C.J. Diez, O. Cabellos, P.E. Labeau, Annals of Nucl. En. 88, 12-23 (2016)

[5] D. Rochman, P. Leray, A. Vasiliev, H. Ferroukhi, A.J. Koning, M. Fleming, J.C. Sublet, Annals of Nucl. En. 95, 125-134 (2016)

[6] O. Serot, C. Amouroux, A. Bidaud, N. Capellan, S. Chabod, A. Ebran, H. Faust, G. Kessedjian, U. Köster, A. Letourneau, O. Litaize, F. Martin, T. Materna, L. Mathieu, S. Panebianco, J.-M. Regis, M. Rudigier, C. Sage, and W. Urban, Nucl. Data Sheets 119, 320-323 (2014)

[7] G. Fioni, H.R. Faust, M. Gross, M. Hesse, P. Armbruster, F. Gönnenwein and G. Müzenberg, Nucl. Instrum. Meth. A 332, 175-180 (1993)

[8] H.R. Faust, P. Geltenbort, F. Gönnenwein and A. Oed, ILL Internal Scientific Report 81FA45S (1981)

[9] M. Schmelling, Physica Scripta 51, 676-679 (1995)

[10] F. Martin, $\mathrm{PhD}$ thesis, Étude des distributions en masse, charge nucléaire et énergie cinétique des produits de fission de $l{ }^{233} U\left(n_{t h}, f\right)$ et du ${ }^{241} P u\left(n_{t h}, f\right)$ mesurées auprès du spectromètre de masse Lohengrin (ILL) (Université de Grenoble, 2013) Chapter 7

[11] S. Julien-Laferrière, ongoing $\mathrm{PhD}$ thesis

[12] G. Knyazheva et al., Nucl. Instrum. Meth. B 7, 248 (2006) 
[13] J.F. Ziegler, M.D. Ziegler and J.P. Biersack, Nucl. Instrum. Meth. B 268, 1818-1823 (2010)

[14] O. Litaize, O. Serot and L. Berge, Eur. Phys. J. A 51, 117 (2015)

[15] C. Wagemans, The Nuclear Fission Process (CRC Press, Boca Raton, Florida, 1991) 319

[16] H. Thierens, E. Jacobs, P. D’Hondt, A. de Clercq, M. Piessens, and D. de Frenne, Phys. Rev. C 29, 498-507 (1984)

[17] J.N. Neiler, F.J. Walter, and H.W. Schmitt, Phys. Rev. 149, 894 (1966) 
